\title{
Narrow band imaging in gastroesophageal reflux disease and Barrett's esophagus
}

\author{
Mitchell M Lee MD ${ }^{1}$, Robert Enns MD FRCPC ${ }^{2}$
}

$\mathrm{N}^{2}$ Tarrow Band Imaging (NBI), developed by Olympus Medical Systems (Olympus, Japan), is a new and wellrecognized advance in endoscopic imaging. Although conventional white-light endoscopy uses the entire spectrum of visible light $(400 \mathrm{~nm}$ to $700 \mathrm{~nm}$ ) to examine tissue, the NBI system uses optic filters to isolate two specific bands of light: $415 \mathrm{~nm}$ blue and $540 \mathrm{~nm}$ green (1-4). By isolating these two bands of light and taking into account their absorptive and reflective properties on the mucosal surface, an image that enhances visualization of superficial mucosal and vascular structures is created. The highlight of NBI endoscopy is the proposed ability to predict pathology in real-time based on the mucosal and vascular changes detected. NBI has been investigated for use as a diagnostic modality in a variety of lesions in the gastrointestinal, respiratory and genitourinary tract (4-6). Conveniently, the NBI mode on an endoscope can be activated with the depression of a switch similar to taking a picture.

NBI, often referred to as 'digital chromoendoscopy', was developed as an alternative method of

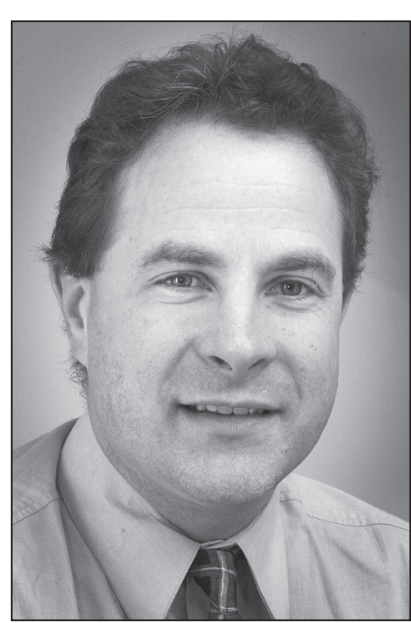

Dr Robert Enns

(NERD), erosive esophagitis and BE (7). Upper endoscopy is considered by many to be a relatively insensitive test for GERD because the disorder often yeilds normal endoscopic findings. Studies (8) have found that fewer than $40 \%$ of patients with GERD have positive upper endoscopy findings. As a result, it obtaining visual enhancement of tissue similar has been hypothesized that the use of NBI endoscopy may help in improving diagnostic accuracy over standard endoscopy in patients with GERD. Most studies investigating the use of NBI endoscopy in GERD have focused on determining the correlation of observed mucosal and vascular patterns with histology from biopsies. Sharma et al (9) found that a significantly higher proportion of patients with GERD had changes in the number $(\mathrm{OR} 12.6 ; \mathrm{P}<0.0001)$, dilation (OR 20; $\mathrm{P}<0.0001)$ and tortuosity (OR 6.9; $\mathrm{P}<0.0001)$ of intrapapillary capillary loops. In addition, the presence of microerosions $(\mathrm{P}<0.0001)$ and an increase in vascularity $(\mathrm{OR}$ 9.3; $\mathrm{P}=0.001)$ at the squamocolumnar junction was noted in patients with GERD compared with controls. A recent study (10) also found that patients with GERD and NERD had changes at the squamocolumnar junction such as the presence of microerosions (GERD 100\%, NERD to that seen in chromoendoscopy - a procedure that uses the absorptive properties of various dyes, such as indigo carmine, rather than optical filters (1-4). Although much research concerning the use of chromoendoscopy has been conducted, its widespread use has not been adopted for various reasons including the increased time involved with the procedure and the lack of training (ie, many training centres do not use it regularly).

In the January 2009 issue of The Canadian Journal of Gastroenterology, we discussed the role of NBI for the detection of neoplastic lesions of the lower gastrointestinal tract; specifically its utility in the diagnosis of adenomatous polyps and dysplasia in ulcerative colitis, hereditary nonpolyposis colorectal cancer and screening colonoscopy. The purpose of the present paper is to review the use of NBI endoscopy as a diagnostic modality for lesions of the upper gastrointestinal tract with particular emphasis on Barrett's esophagus (BE).

\section{NBI ENDOSCOPY IN GASTROESOPHAGEAL REFLUX DISEASE}

Presently, few studies in the literature have investigated the use of NBI for the diagnosis or management of gastroesophageal reflux disease (GERD). Based on clinical history and upper endoscopy findings, patients with GERD are typically classified into the following groups: nonerosive reflux disease $52.8 \%$, controls $23.3 \%$ ) and an increase in vascularity (GERD 95.1\%, NERD 91.7\%, controls 36.7\%).

To date, the research performed concerning the use of NBI endoscopy in GERD is relatively new, and there is still a need for prospective randomized controlled trials to validate the mucosal and vascular patterns seen in the above studies. Furthermore, additional research is needed to fully assess the clinical utility of NBI, not only in the diagnosis of GERD compared with conventional endoscopy, but also how it will possibly change the management of patients if they are diagnosed with mild erosive disease.

\section{NBI ENDOSCOPY IN BE}

$\mathrm{BE}$ is defined histologically by the replacement of the normal squamous epithelium of the esophagus with columnar-lined epithelium (CLE) and specialized intestinal metaplasia (SIM) $(11,12)$. GERD is a major risk factor for the development of $\mathrm{BE}$ because it is believed that chronic acid reflux causes injury to the squamous epithelium of the esophagus, leading to the mucosal changes associated with $\mathrm{BE}(13)$. BE is of clinical importance because it is considered to be a premalignant lesion of esophageal adenocarcinoma $(14,15)$. In patients with BE, current protocols recommend upper endoscopy surveillance with four-quadrant random biopsies at $2 \mathrm{~cm}$ intervals of

${ }^{1}$ University of British Columbia, Faculty of Medicine; ${ }^{2}$ St Paul's Hospital, Division of Gastroenterology, Vancouver, British Columbia

Correspondence: Dr Robert Enns, St Paul's Hospital, University of British Columbia, Pacific Gastroenterology Associates, 770-1190 Hornby Street,

Vancouver, British Columbia V6Z 2K5. Telephone 604-689-6332, fax 604-689-2004, e-mail renns@interchange.ubc.ca

Received and accepted for publication December 30, 2008 
Barrett's epithelium and targeted biopsies of abnormal mucosa to detect neoplastic changes (16-19). The incidence of esophageal adenocarcinoma is at least 30-fold higher in patients with BE compared with the general population $(11,20)$. Despite the increasing incidence of esophageal adenocarcinoma in Europe and North America, it is often difficult to detect the subtle, early dysplastic appearing lesions in $\operatorname{BE}(21,22)$. As a result, a number of new technologies have been proposed for use in the diagnosis and surveillance of patients with $\mathrm{BE}$ including high-resolution white-light endoscopy, autofluorescence imaging, chromoendoscopy and NBI to improve detection rates $(11,23-28)$ of dysplasia. NBI also has the benefit of allowing the endoscopist to make an in vivo diagnosis, without the need for random biopsies, which can theoretically be costly, time consuming and can occasionally lead to sampling errors $(29,30)$. It is hoped that with improvements in the diagnosis of $\mathrm{BE}$ and its complications, early intervention can be undertaken to improve patient outcomes. Early intervention is available at many centres with the development of endoscopic mucosal resections and several ablative techniques; it is possible that more aggressive therapy of earlier lesions will be advocated.

\section{Characterization of lesions seen in $\mathrm{BE}$ with NBI endoscopy}

A number of studies (31-35) have described the spectrum of changes from CLE to dysplasia, seen with NBI endoscopy in BE. Kara et al (31) used a classification scheme for $\mathrm{BE}$ which focused on three criteria: the mucosal pattern (flat, villous/gyrus, other, irregular/disrupted), the vascular pattern (regular, irregular, normal-appearing longbranched) and the presence of abnormal blood vessels. SIM was characterized by villous/gyrus mucosal patterns $(80 \%$ of cases) and flat mucosa with normalappearing long-branched vessels $(20 \%$ of cases). High-grade intraepithelial neoplasia was characterized by three patterns: irregular/disrupted mucosal patterns, irregular vascular patterns and abnormal blood vessels. A study by Sharma et al (32) in 2006 classified NBI images according to mucosal (ridge/villous, circular, irregular) and vascular (normal and abnormal) patterns. The NBI images, correlated with histology, found that the ridge/villous pattern had a sensitivity, specificity and positive predictive value (PPV) of $93.5 \%, 85.7 \%$ and $94.7 \%$, respectively, for the diagnosis of SIM. The irregular/ distorted vascular pattern had a sensitivity, specificity and PPV of $100 \%, 98.7 \%$, and $95.3 \%$, respectively, for high-grade dysplasia. Goda et al (33) classified the mucosal (1 round/oval, 2 long straight, 3 villous, 4 cerebriform and 5 irregular) and vascular (I honeycomb-like, II vinelike, III coiled, IV ivy-like and V irregular) patterns seen with magnifying endoscopy under NBI for the detection of SIM and superficial Barrett's adenocarcinoma. The most characteristic endoscopic patterns to suggest Barrett's epithelium were the cerebriform mucosal pattern (sensitivity $56 \%$, specificity $79 \%$, OR 4.78) and the ivy-like capillary pattern (sensitivity $77 \%$, specificity 94\%, OR 51.6). A study in 2007 (34) investigated the endoscopic features seen in BE under magnified endoscopy with NBI, using a classification scheme consisting of microstructural (regular, irregular, absent) and microvascular (regular and irregular) patterns. The finding of regular microstructural pattern with tubular/linear/villous pattern and regular microvascular pattern with absent microstructural pattern had a diagnostic yield of $90.6 \%$ and $98.9 \%$ respectively, for SIM. The irregular microvascular/microstructural pattern had a sensitivity and specificity of $90 \%$ and $100 \%$, respectively, for the prediction of high-grade dysplasia. Recently, Singh et al (35) proposed a classification scheme using four common patterns found with NBI endoscopic examination in patients with BE: pattern A (round pits with regular microvasculature), pattern $B$ (villous/ridge pits with regular microvasculature), pattern C (absent pits with regular microvasculature) and pattern $\mathrm{D}$ (distorted pits with irregular microvasculature). Pattern A had a PPV and negative predictive value (NPV) of $100 \%$ and $97 \%$, respectively, for columnar mucosa without intestinal metaplasia. Patterns B and C had a PPV and NPV of $88 \%$ and $91 \%$, respectively, for specialized intestinal metaplasia. Pattern D had a PPV and NPV of $81 \%$ and $99 \%$, respectively, for high-grade dysplasia.

Although the available results from the studies describing the changes seen under NBI endoscopy for BE appear promising, it is important to note that most groups developed their own classification 
system for the mucosal and vascular changes observed. As a result, the lack of a sufficiently validated and standardized classification scheme is one of the main limitations in the use of NBI for BE. With the results of studies characterizing the lesions seen under $\mathrm{NBI}$, the next step in investigating this modality is to validate the classification schemes described above, and to determine its clinical utility by comparing it with conventional white-light endoscopy and other modalities such as chromoendoscopy. We will discuss these studies in the next section.

\section{NBI ENDOSCOPY VERSUS CONVENTIONAL ENDOSCOPY IN BE}

A number of studies have investigated measures such as sensitivity, specificity, diagnostic accuracy and image quality of the NBI system compared with conventional white-light endoscopy in BE. A study in 2004 by Hamamoto et al (11) investigated conventional white-light magnifying endoscopy versus NBI endoscopy in 11 patients with BE. Images taken during the procedure were reviewed with a scoring system ( 0 to 4 , with 4 being optimal) to assess the quality of images of the esophagogastric junction, capillary vessels and CLE. The squamocolumnar junction was visualized with a score of 3 or greater in $57 \%$ and $17 \%(\mathrm{P}=0.0002)$ of images under NBI and conventional endoscopy, respectively, while net-like blood vessels were visualized with a score of 3 or greater in $68 \%$ and $42 \%$ $(\mathrm{P}=0.1919)$ of images for NBI and conventional endoscopy, respectively. The CLE was visualized with a score of 3 or greater in $100 \%$ and $80 \%(\mathrm{P}=0.11)$ of lesions for NBI and conventional endoscopy, respectively. Although there are limitations in assessing images for subjective factors such as image quality, this paper showed that NBI improves the visualization of important structures seen in BE over conventional endoscopy.

Curvers et al (23) compared high-resolution white-light endoscopy with NBI and determined that the yield of early neoplasia was $81 \%, 72 \%$ and $83 \%$ in the white-light endoscopy, NBI, and combination (white-light endoscopy and NBI) groups, respectively. Interestingly, the image quality was significantly better in the NBI group over the white-light endoscopy group ( 11.3 versus 10.9 on a visual analogue scale; $\mathrm{P}=0.01$ ) but was not found to improve the diagnostic yield of neoplasia. Another study by Singh et al (24) compared the use of highresolution magnified NBI with conventional white-light endoscopy with magnification in 21 patients with BE and found that the sensitivity, specificity, and accuracy of NBI with magnification was $88.9 \%, 94.8 \%$ and $91.3 \%$, respectively, compared with $71.9 \%, 90.5 \%$ and $84.8 \%$ for conventional white-light endoscopy for the prediction of histology. When both modalities were combined, a sensitivity, specificity and accuracy of $90.2 \%, 95 \%$ and $91.7 \%$ respectively, was achieved. Furthermore, in this study supporting the use of NBI there was a significant difference between NBI and conventional endoscopy for the detection of high-grade dysplasia (95\% versus $62.5 \%$; $\mathrm{P}<0.006$ ).

Wolfsen et al (25) investigated whether NBI targeted biopsies could detect advanced dysplasia using fewer biopsy samples

\section{REFERENCES}

1. Gono K, Obi T, Yamaguchi M, et al. Appearance of enhanced tissue features in narrow-band endoscopic imaging. J Biomed Opt 2004;9:568-77.

2. Yoshida T, Inoue H, Usui S, et al. Narrow-band imaging system with magnifying endoscopy for superficial esophageal lesions. Gastrointest Endosc 2004;59:288-95. compared with conventional endoscopy using the four-quadrant biopsy method with a prospective, blinded, controlled tandem study. The study revealed that NBI detected dysplasia in $57 \%$ of patients compared with $43 \%$ in the conventional endoscopy with four-quadrant biopsy group, with higher grades of dysplasia detected in the NBI group $(\mathrm{P}<0.001)$. In addition, more biopsies were taken in the four-quadrant biopsy group compared with narrow-band targeted biopsies (mean 8.5 versus 4.7; $\mathrm{P}<0.001$ ). Consequently, the use of 'targeted' biopsy techniques have potential time and cost savings.

Studies have also investigated the use of NBI compared with chromoendoscopy in BE. Curvers et al (26) recently investigated chromoendoscopy, NBI and high-resolution magnification endoscopy in patients with $\mathrm{BE}$. They found that high-resolution endoscopy alone had an $86 \%$ yield of identifying high-grade dysplasia, while the combinations of white-light endoscopy with NBI, indigo carmine chromoendoscopy and acetic acid chromoendoscopy had $84 \%, 70 \%$ and $83 \%$ yields, respectively. A study by Kara et al (27) in 2005 investigated chromoendoscopy versus NBI, both in combination with highresolution endoscopy, in a prospective, randomized crossover study with 14 patients. The sensitivity of chromoendoscopy and NBI was $93 \%$ and $86 \%$, respectively, compared with $79 \%$ for four-quadrant biopsies with conventional endoscopy in the diagnosis of high-grade dysplasia or early cancer in patients with BE.

Although the results of studies comparing the use of NBI endoscopy with other modalities appear favourable, further randomized controlled studies comparing NBI with conventional endoscopy with the four-quadrant protocol for surveillance are required before further recommendations can be made regarding the use of NBI in routine practice. Presently, NBI is typically used as an adjunct to conventional white-light endoscopy to investigate areas appearing suspicious after initial screening.

\section{CONCLUSION}

We have reviewed the current literature regarding the utility of NBI in the upper gastrointestinal tract, with particular emphasis on BE. Although other areas of the upper gastrointestinal tract (ie, stomach in the setting of intestinal metaplasia) may have lesions at increased risk and benefit from NBI, most studies have been performed in the setting of BE. Although there are limited numbers of studies regarding the use of NBI in the upper gastrointestinal tract, the available research appears promising. Presently, NBI is used as an adjunct to conventional white-light endoscopy for targeted investigation of suspicious areas. The main limitations of the NBI system include the learning curve associated with the new technology, the lack of sufficiently validated and standardized classification schemes for the NBI patterns observed in various conditions, and the limited number of randomized controlled trials investigating NBI compared with conventional white-light endoscopy. Further large-scale studies are required to address these limitations before NBI can be recommended as a primary method of screening for lesions of the esophagus in routine practice.

3. Sano Y, Muto M, Tajiri H, et al. Optical/digital chromoendoscopy during colonoscopy using narrow-band imaging system. Dig Endosc 2005;S43-8.

4. ASGE Technology Committee, Song LM, Adler DG, Conway JD, et al. Narrow band imaging and multiband imaging. Gastrointest Endosc 2008;67:581-9. 
5. Watanabe A, Taniguchi M, Tsujie $\mathrm{H}$, et al. The value of narrow band imaging for early detection of laryngeal cancer. Eur Arch Otorhinolaryngol 2008. [Epub ahead of print]

6. Herr HW, Donat SM. A comparison of white-light cystoscopy and narrow-band imaging cystoscopy to detect bladder tumour recurrences. BJU Int 2008;102:1111-4

7. Fass R, Ofman JJ. Gastroesophageal reflux disease - should we adopt a new conceptual framework? Am J Gastrointerol 2002;97:1901-9.

8. Dent J, Armstrong D, Delaney B, et al. Symptom evaluation in reflux disease: Workshop background, processes, terminology, recommendations, and discussion outputs. Gut 2004:53(Suppl 4):1-24.

9. Sharma P, Wani S, Bansal A, et al. A feasibility trial of narrow band imaging endoscopy in patients with gastroesophageal reflux disease. Gastroenterology 2007;133:454-64.

10. Fock KM, Teo EK, Ang TL, et al. The utility of narrow band imaging in improving the endoscopic diagnosis of gastroesophageal reflux disease. Clin Gastroenterol Heptatol 2008; [Epub ahead of print].

11. Hamamoto Y, Endo T, Nosho K, et al. Usefulness of narrow-band imaging endoscopy for diagnosis of Barrett's esophagus. J Gastroenterol 2004;39:14-20.

12. Spechler SJ. Clinical practice. Barrett's esophagus. N Engl J Med 2002;346:836-42.

13. Spechler SJ. Barrett's esophagus and esophageal adenocarcinoma: Pathogenesis, diagnosis, and therapy. Med Clin North Am 2002;86:1423-45.

14. Haggitt R. Barrett's esophagus, dysplasia, and adenocarcinoma. Hum Pathol 1994;25:982-93.

15. Sharma P, McQuaid K, Dent J, et al. A critical review of the diagnosis and management of Barrett's esophagus: The AGA Chicago Workshop. Gastroenterology 2004;127:310-30

16. Spechler SJ. Barrett's esophagus. Gastroenterologist 1994;2:273-84

17. Sampliner RE. Practice guidelines on the diagnosis, surveillance, and therapy of Barrett's esophagus: The Practice Parameters Committee of the American College of Gastrointerology. Am J Gastroenterol 1999;94:1434-42.

18. Hirota WK, Zuckerman MJ, Adler DG, et al. ASGE guideline: The role of endoscopy in the surveillance of premalignant conditions of the upper GI tract. Gastrointest Endosc 2006;63:570-80.

19. Reid BJ, Weinstein WM, Lewin KJ, et al. Endoscopic biopsy can detect high-grade dysplasia or early adenocarcinoma in Barrett's esophagus without grossly recognized neoplastic lesions. Gastroenterology 1988;94:81-90.

20. Souza RF, Spechler SJ. Concepts in the prevention of adenocarcinoma of the distal esophagus and proximal stomach. CA Cancer J Clin 2005;55:334-51.

21. Devesa SS, Blot WJ, Fraumeni JF Jr. Changing patterns in the incidence of esophageal and gastric carcinoma in the United States. Cancer 1998;83:2049-53.

22. Blots WJ, Devesa SS, Kneller RW, et al. Rising incidence of adenocarcinoma of the esophagus and gastric cardia. JAMA 1991;256:1287-9.

23. Curvers WL, Bohmer CJ, Mallant-Hent RC, et al. Mucosal morphology in Barrett's esophagus: Interobserver agreement and role of narrow band imaging. Endoscopy 2008;40:799-805.

24. Singh R, Karageorgiou H, Owen V, et al. Comparison of high-resolution magnification narrow-band imaging and white-light endoscopy in the prediction of histology in Barrett's esophagus. Scand J Gastroenterol 2008;26:1-8.

25. Wolfsen HC, Crook JE, Krishna M, et al. Prospective, controlled tandem endoscopy study of narrow band imaging for dysplasia detection in Barrett's esophagus. Gastroenterology 2008;135:24-31.

26. Curvers W, Baak L, Kiesslich R, et al. Chromoendoscopy and narrow-band imaging compared with high-resolution magnification endoscopy in Barrett's esophagus. Gastroenterology 2008;134:670-9.

27. Kara MA, Peters FP, Rosmolen WD, et al. High-resolution endoscopy plus chromoendoscopy or narrow-band imaging in Barrett's esophagus: A prospective randomized crossover study. Endoscopy 2005;37:929-36.

28. Kara MA, Bergman JJ. Autofluorescence imaging and narrow-band imaging for the detection of early neoplasia in patients with Barrett's esophagus. Endoscopy 2006;38:627-31

29. Spechler SJ, Goyal RK. The columnar-lined esophagus, intestinal metaplasia, and Norman Barrett. Gastroenterology 1996;110:614-21.

30. Wani S, Sharma P. Endoscopic surface imaging of Barrett's esophagus: An optimistic view. Gastroenterology 2007;133:11-13.

31. Kara MA, Ennahachi M, Fockens P, et al. Detection and classification of the mucosal and vascular patterns (mucosal morphology) in Barrett's esophagus by using narrow band imaging. Gastrointest Endosc 2006;64:155-66.

32. Sharma P, Bansal A, Mathur S, et al. The utility of a novel narrow band imaging endoscopy system in patients with Barrett's esophagus. Gastrointest Endosc 2006;64:167-75.

33. Goda K, Tajiri H, Ikegami M, et al. Usefulness of magnifying endoscopy with narrow band imaging for the detection of specialized intestinal metaplasia in columnar-lined esophagus and Barrett's adenocarcinoma. Gastrointest Endosc 2007;65:36-46

34. Anagnostopoulos GK, Yao K, Kaye P, et al. Novel endoscopic observation in Barrett's oesophagus using high resolution magnification endoscopy and narrow band imaging. Aliment Pharmacol Ther 2007;26:501-7.

35. Singh R, Anagnostopoulos GK, Yao K, et al. Narrow-Band imaging with magnification in Barrett's esophagus: Validation of a simplified grading system of mucosal morphology patterns against histology. Endoscopy 2008;40:457-63. 


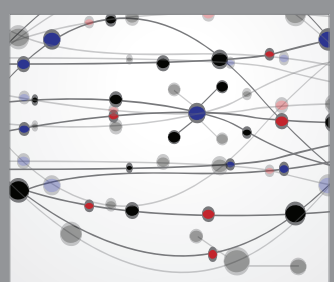

The Scientific World Journal
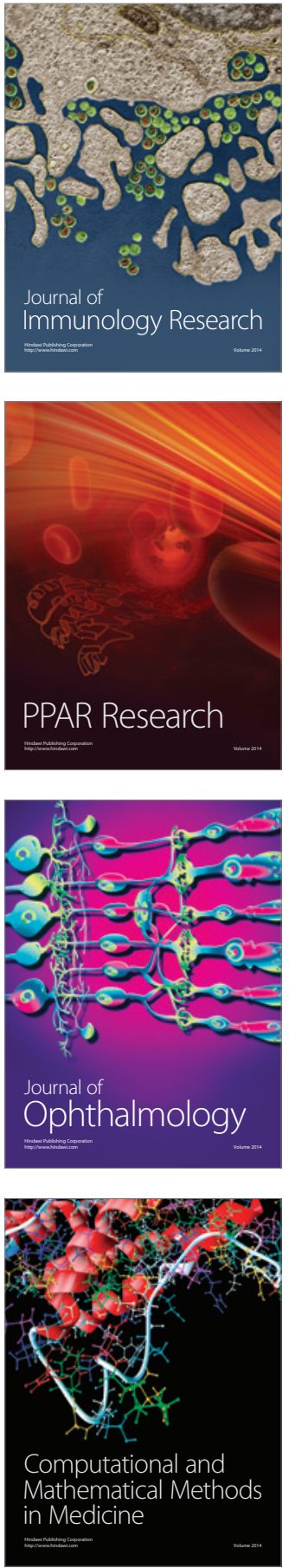

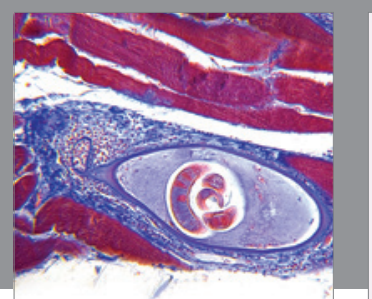

Gastroenterology Research and Practice

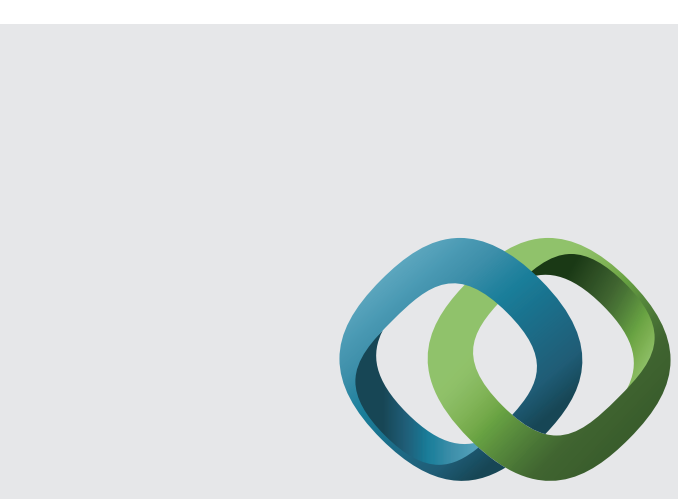

\section{Hindawi}

Submit your manuscripts at

http://www.hindawi.com
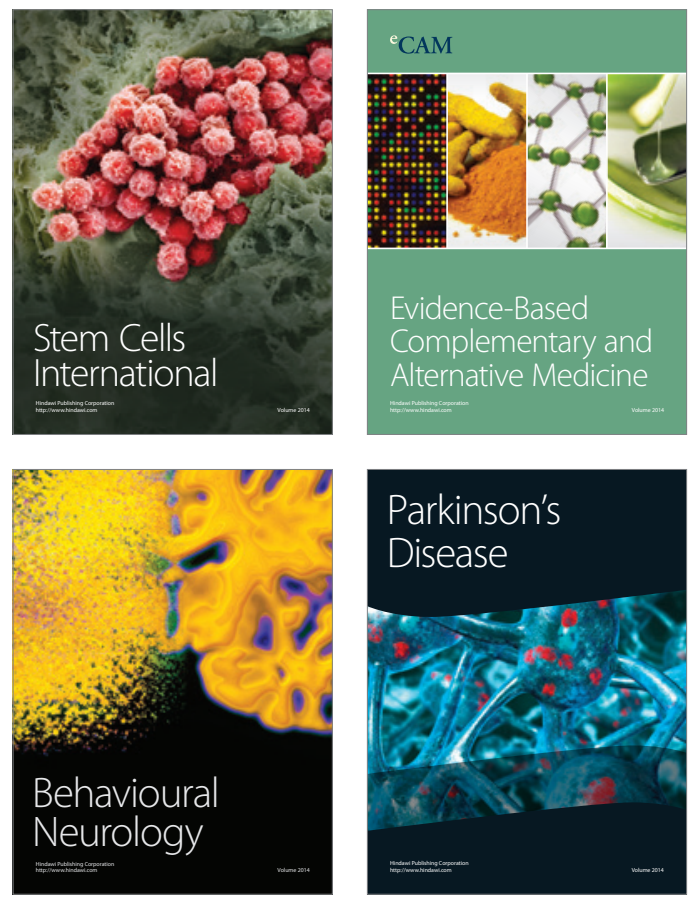
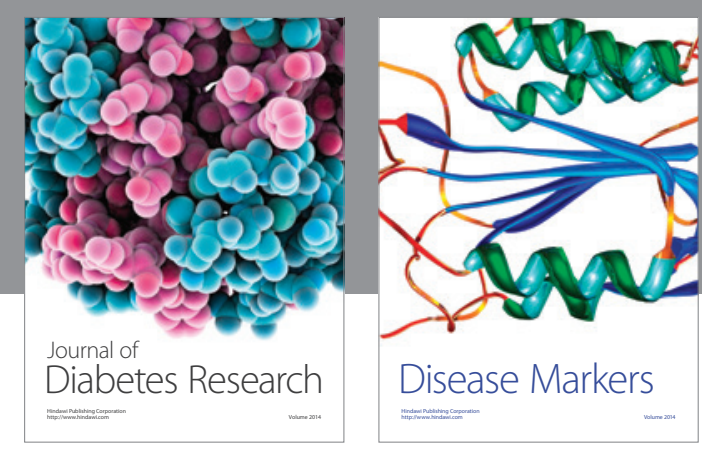

Disease Markers
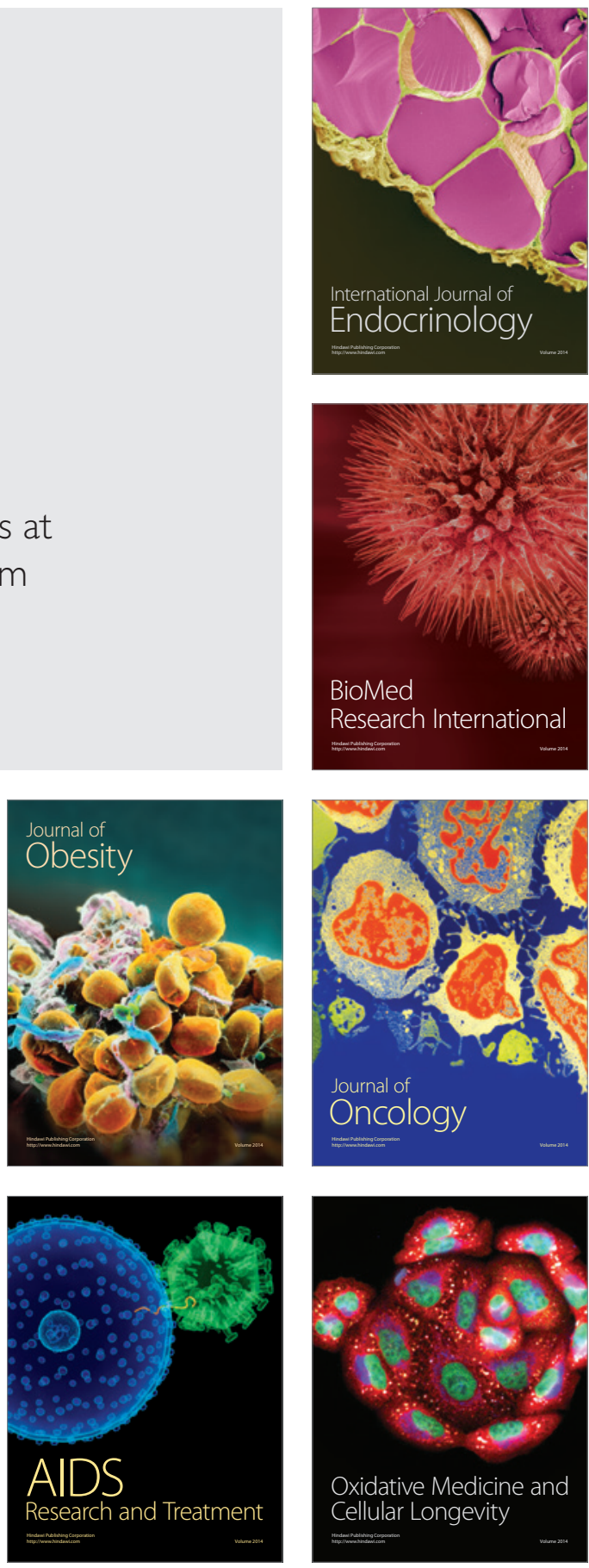